\title{
Association between serum creatinine level within the normal range and bone mineral density in adolescents
}

\author{
Kaiyu Pan \\ Hangzhou Xiaoshan No 1 People's Hospital \\ Haiyan Yu \\ Hangzhou Xiaoshan No 1 People's Hospital \\ Zhongxin Zhu ( $D$ orthozzx@163.com) \\ Hangzhou Xiaoshan No 1 People's Hospital https://orcid.org/0000-0002-5924-6748
}

\section{Research article}

Keywords: creatinine, bone health, adolescent, biomarker, NHANES

Posted Date: April 8th, 2021

DOl: https://doi.org/10.21203/rs.3.rs-376819/v1

License: (c) (i) This work is licensed under a Creative Commons Attribution 4.0 International License.

Read Full License 


\section{Abstract}

Objective: The circulating level of creatinine is a direct, stable indicator of skeletal muscle mass. However, evidence regarding the correlation between serum creatinine (SCre) and bone health is limited. This study aimed to evaluate the association between SCre level within the normal range and bone mineral density (BMD) in adolescents.

Methods: We analyzed data for 3456 adolescents aged 12-19 years from the National Health and Nutrition Examination Survey 2011-2018. Weighted multiple linear regression was conducted to assess the association between SCre and BMD. Weighted generalised additive models and smooth curve fittings were performed to address the nonlinearity of them.

Results: After controlling for potential confounding factors, we found that higher SCre levels were associated with higher total BMD in adolescents. This association remained positive in the subgroup analyses stratified by age, gender, or race. Furthermore, this positive association was more prominent in boys than in girls in adolescents aged 12-15 years.

Conclusions: These findings indicate that higher SCre levels within the normal range in adolescents aged 12-19 years were associated with higher total BMDs, suggesting that SCre may be a candidate biomarker for bone health in adolescents.

\section{Introduction}

Osteoporosis has been recognized as a disease during the aging process, but it is now widely accepted that it begins from childhood and adolescence by lack of bone mineral density (BMD) built during these periods[1]. Bone mass reaches the maximum of $90 \%$ at adolescence and slightly increases after this period $[2,3]$. Therefore, it is critical to acquire a higher BMD during adolescence and thus prevent osteoporosis and osteoporosis-associated fractures in older age.

To better understand the pathogenesis of osteoporosis, ongoing studies are exploring the associations between bone health and some novel or less studied markers[4]. In clinical practice, creatinine-based estimated glomerular filtration rate is widely used as a measure of renal function[5]. Creatine is a naturally occurring guanidino compound composed of arginine and glycine, with $>90 \%$ of total body creatine being stored within skeletal muscle[6]. The irreversible process that creatinine is nonenzymatically converted from creatine results in a turnover of $\approx 1.7 \%$ of the total creatine daily[7]. The circulating level of creatinine is a direct, stable indicator of skeletal muscle mass because its generation is proportional to muscle mass[8]. However, evidence regarding the correlation between serum creatinine (SCre) and bone health is limited. A recent general population-based study revealed that low SCre was independently associated with low BMD in participants aged 45-95 years with normal renal function[9]. However, to our knowledge no previous studies have examined the association between SCre and BMD in adolescents. Therefore, we conducted a cross-sectional study of a subsample of 3456 adolescents aged 12-19 years participating in the National Health and Nutrition Examination Survey (NHANES) from 2011 
to 2018. In our analysis, we considered and tested potential confounding factors, including age, gender, and race.

\section{Methods}

\section{Study population}

NHANES is a program of studies that is designed to assess the health and nutrition status of US population. We utilized data from NHANES collected from 2011 to 2018. Based on age, gender, and race, NHANES uses a complex, stratified, multistage probability sampling designed to be representative of the civilian, non-institutionalized US population. The ethics review board of the National Center for Health Statistics (NCHS) approved all NHANES protocols. Participants aged $\geq 18$ years provided informed consent, and for participants aged $<18$ years, their parents/guardians provided informed consent.

Our sample included adolescents aged 12-19 years with complete data for SCre and total BMD ( $\mathrm{n}=$ 3749). Additionally, we further excluded 293 subjects whose SCre levels were not within the normal range[10], resulting in 3456 subjects remained for the final analysis (Fig. 1).

\section{Study variables}

The concentration of SCre was determined on the Beckman DXC800 analyzer using the Jaffe rate method (kinetic alkaline picrate). According to the laboratory procedure manual, the normal range was 0.3-1.0 mg/dL for adolescents aged $12-15$ years, $0.7-1.3 \mathrm{mg} / \mathrm{dL}$ for boys aged $16-19$ years, and 0.6$1.1 \mathrm{mg} / \mathrm{dL}$ for girls aged 16-19 years (we used the International System of Units in this study, and converted $\mathrm{mg} / \mathrm{dL}$ to umol/L by multiplying by 88.4 [ [10]. The whole body scans were acquired on the Hologic Discovery model A densitometers, using software version Apex 3.2. To verify the accuracy and consistency of the results, expert review was conducted on $100 \%$ of analyzed participant scans[11].

Additionally, we included following covariates: age, gender, race, body mass index (BMI), ratio of family income to poverty, vigorous recreational activities, total cholesterol, total protein, blood urea nitrogen (BUN), serum glycohemoglobin, alkaline phosphatase (ALP), alanine amino transferase (ALT), aspartic acid transferase (AST), gamma-glutamyl transpeptidase (GGT), serum uric acid (sUA), serum phosphorus, and serum calcium were adjusted. Details of the acquisition process of SCre, total BMD and other covariate are available at www.cdc.gov/nchs/nhanes/.

\section{Statistical analysis}

To account for the probabilities of selection and response as well as total US population, all estimates were calculated using sampling weights as recommended by NCHS. Weighted multiple regression analysis was conducted to evaluate the independent association between SCre and BMD. Following the STROBE guidelines[12], three weighted multivariate linear regression models were constructed: model 0 : not adjusted; model 1: age, gender, race were adjusted; model 2: the covariates presented in Table 1 were 
adjusted. Weighted generalised additive models and smooth curve fittings were performed to address the nonlinearity of SCre and BMD. All analyses were done by using EmpowerStats software and R version 3.4.3. $\mathrm{P}<0.05$ was considered statistically significant. 
Table 1

Weighted characteristic of study sample.

\begin{tabular}{|c|c|c|c|}
\hline & Boys $(n=1882)$ & Girls $(n=1574)$ & $P$ value \\
\hline Age (years) & $15.36 \pm 2.30$ & $15.28 \pm 2.21$ & 0.319 \\
\hline Age groups (\%) & & & 0.570 \\
\hline $12-15$ years & 53.55 & 54.52 & \\
\hline $16-19$ years & 46.45 & 45.48 & \\
\hline Race (\%) & & & 0.447 \\
\hline Non-Hispanic White & 54.99 & 56.73 & \\
\hline Non-Hispanic Black & 12.83 & 12.81 & \\
\hline Mexican American & 15.65 & 13.72 & \\
\hline Other Race & 16.53 & 16.74 & \\
\hline Body mass index $(\mathrm{kg} / \mathrm{m} 2)$ & $23.61 \pm 5.76$ & $24.31 \pm 6.06$ & $<0.001$ \\
\hline Ratio of family income to poverty & $2.51 \pm 1.60$ & $2.47 \pm 1.62$ & 0.528 \\
\hline Vigorous recreational activities (\%) & & & $<0.001$ \\
\hline Yes & 58.79 & 41.70 & \\
\hline No & 24.42 & 41.23 & \\
\hline Not recorded & 16.79 & 17.07 & \\
\hline Blood urea nitrogen $((\mathrm{mmol} / \mathrm{L})$ & $4.30 \pm 1.24$ & $3.73 \pm 1.06$ & $<0.001$ \\
\hline Total protein $(\mathrm{g} / \mathrm{L})$ & $72.47 \pm 4.12$ & $72.01 \pm 4.04$ & $<0.001$ \\
\hline Total cholesterol ((mmol/L) & $3.94 \pm 0.72$ & $4.12 \pm 0.78$ & $<0.001$ \\
\hline Serum glycohemoglobin (\%) & $5.23 \pm 0.34$ & $5.23 \pm 0.36$ & 0.860 \\
\hline Alkaline phosphatase (U/L) & $174.68 \pm 106.68$ & $101.28 \pm 62.65$ & $<0.001$ \\
\hline Alanine amino transferase (IU/L) & $20.94 \pm 13.58$ & $15.89 \pm 12.94$ & $<0.001$ \\
\hline Aspartic acid transferase (IU/L) & $25.35 \pm 13.26$ & $20.82 \pm 7.44$ & $<0.001$ \\
\hline Gamma-glutamyl transpeptidase (IU/L) & $15.95 \pm 9.54$ & $12.32 \pm 5.66$ & $<0.001$ \\
\hline Serum uric acid(umol/L) & $333.50 \pm 68.54$ & $266.81 \pm 58.30$ & $<0.001$ \\
\hline
\end{tabular}

Mean \pm SD for continuous variables: $P$ value was calculated by weighted linear regression model. $\%$ for categorical variables: P value was calculated by weighted chi-square test. 


\begin{tabular}{|llll|}
\hline & Boys $(\mathbf{n = 1 8 8 2})$ & Girls $(\mathbf{n = 1 5 7 4 )}$ & P value \\
\hline Serum phosphorus $(\mathrm{mmol} / \mathrm{L})$ & $1.44 \pm 0.23$ & $1.35 \pm 0.18$ & $<0.001$ \\
\hline Serum calcium $(\mathrm{mmol} / \mathrm{L})$ & $2.41 \pm 0.07$ & $2.38 \pm 0.08$ & $<0.001$ \\
\hline Serum creatinine $(\mathrm{umol} / \mathrm{L})$ & $69.02 \pm 15.37$ & $59.23 \pm 10.40$ & $<0.001$ \\
\hline Total bone mineral density $\left(\mathrm{g} / \mathrm{cm}^{2}\right)$ & $1.04 \pm 0.14$ & $1.02 \pm 0.10$ & $<0.001$ \\
\hline Mean \pm SD for continuous variables: P value was calculated by weighted linear regression model. \\
\hline \% for categorical variables: $P$ value was calculated by weighted chi-square test. & \\
\hline
\end{tabular}

\section{Results}

The weighted characteristics of the participants are presented in Table 1. A total of 1882 boys and 1574 girls were included in this study. Among different gender groups, BMI, vigorous recreational activities, BUN, total protein, total cholesterol, ALP, ALT, AST, GGT, sUA, serum phosphorus, serum calcium, SCre, and total BMD were significantly different.

In the weighted multiple regression (Table 2), higher SCre levels were associated with higher total BMDs in each model [model 0: $0.0052(0.0050,0.0054)$; model 1: $0.0036(0.0033,0.0039)$; model 2: 0.0029 $(0.0026,0.0032)]$. 
Table 2

Association between serum creatinine (umol/L) and total bone mineral density $\left(\mathrm{g} / \mathrm{cm}^{2}\right)$.

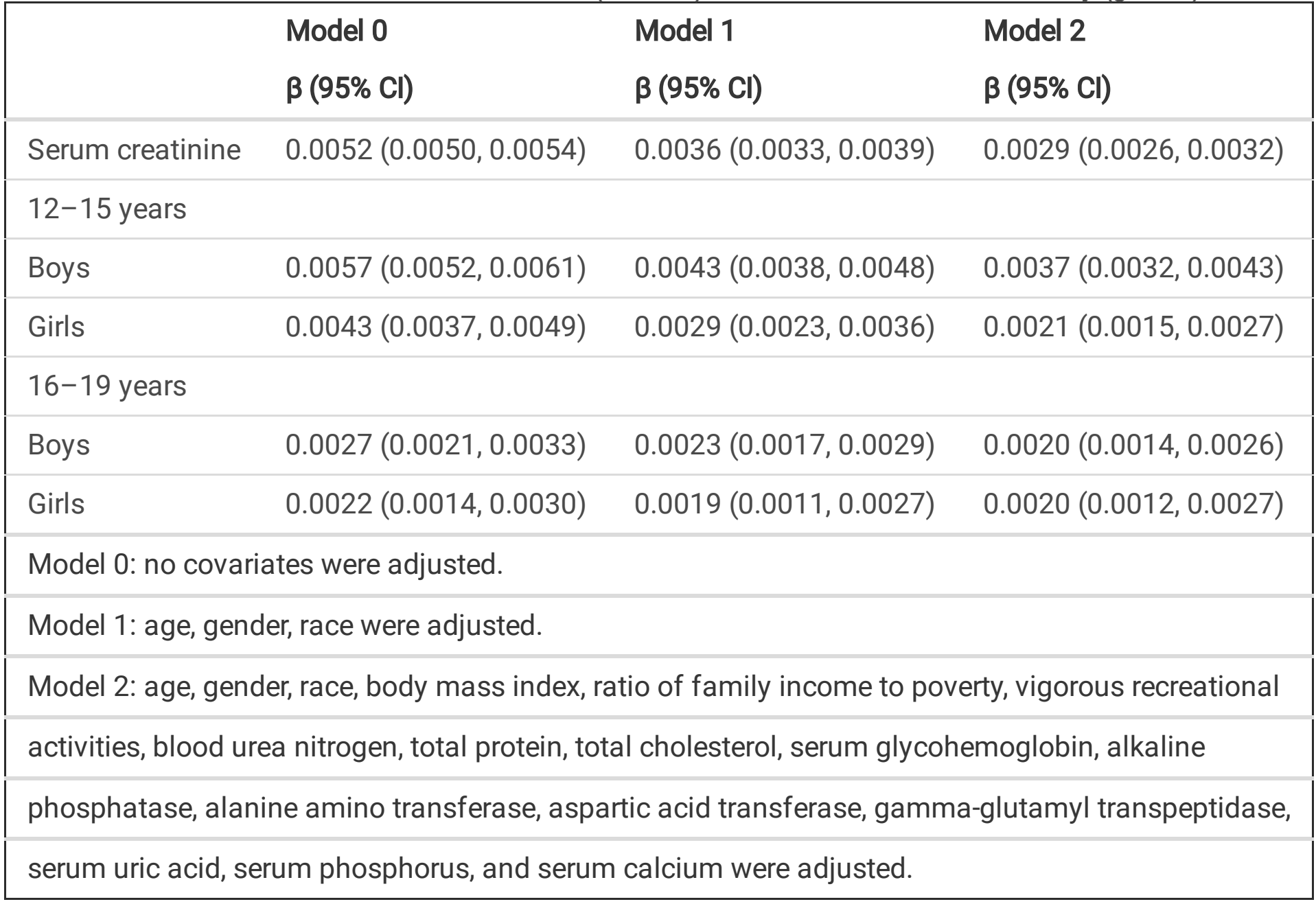

In the subgroup analyses stratified by age and gender (Table 2), the positive association between SCre and total BMD remained in each group after controling for potential confounders [boys aged 12-15 years: $0.0037(0.0032,0.0043)$; girls aged $12-15$ years: $0.0021(0.0015,0.0027)$; boys aged $16-19$ years: $0.0020(0.0014,0.0026)$; girls aged $16-19$ years: $0.0020(0.0012,0.0027)]$.

In the subgroup analyses stratified by age and race (Table 3), this association remained positive in each model of all groups. 
Table 3

Associations between serum creatinine (umol/L) and total bone mineral density $\left(\mathrm{g} / \mathrm{cm}^{2}\right)$, stratified by race.

\begin{tabular}{|c|c|c|c|}
\hline & Model 0 & Model 1 & Model 2 \\
\hline & $\beta(95 \% \mathrm{Cl})$ & $\beta(95 \% \mathrm{Cl})$ & $\beta(95 \% \mathrm{Cl})$ \\
\hline $12-15$ years & & & \\
\hline $\begin{array}{l}\text { Non-Hispanic } \\
\text { White }\end{array}$ & $\begin{array}{l}0.0048(0.0040 \\
0.0055)\end{array}$ & $0.0038(0.0030,0.0046)$ & $0.0034(0.0026,0.0042)$ \\
\hline Non-Hispanic Black & $\begin{array}{l}0.0046(0.0038, \\
0.0054)\end{array}$ & $0.0037(0.0029,0.0045)$ & $0.0026(0.0017,0.0034)$ \\
\hline Mexican American & $\begin{array}{l}0.0046(0.0038 \\
0.0054)\end{array}$ & $0.0037(0.0027,0.0046)$ & $0.0036(0.0027,0.0046)$ \\
\hline Other Race & $\begin{array}{l}0.0047(0.0041 \\
0.0054)\end{array}$ & $0.0037(0.0031,0.0044)$ & $0.0028(0.0021,0.0035)$ \\
\hline $16-19$ years & & & \\
\hline $\begin{array}{l}\text { Non-Hispanic } \\
\text { White }\end{array}$ & $\begin{array}{l}0.0028(0.0021, \\
0.0034)\end{array}$ & $0.0020(0.0012,0.0028)$ & $0.0020(0.0012,0.0028)$ \\
\hline Non-Hispanic Black & $\begin{array}{l}0.0031(0.0024 \\
0.0038)\end{array}$ & $0.0024(0.0015,0.0034)$ & $0.0015(0.0005,0.0025)$ \\
\hline Mexican American & $\begin{array}{l}0.0029(0.0020 \\
0.0039)\end{array}$ & $0.0018(0.0007,0.0030)$ & $0.0020(0.0009,0.0031)$ \\
\hline Other Race & $\begin{array}{l}0.0035(0.0027 \\
0.0043)\end{array}$ & $0.0030(0.0019,0.0040)$ & $0.0025(0.0015,0.0036)$ \\
\hline Model 0: no covari & s were adjusted. & & \\
\hline Model 1: age, gende & were adjusted. & & \\
\hline $\begin{array}{l}\text { Model 2: age, gende } \\
\text { activities, blood urea } \\
\text { phosphatase, alanin } \\
\text { serum uric acid, seru }\end{array}$ & $\begin{array}{l}\text { ody mass index } \\
\text { trogen, total prc } \\
\text { imino transfera } \\
\text { phosphorus, ar }\end{array}$ & $\begin{array}{l}\text { mily income to poverty, } \\
\text { holesterol, serum glycoh } \\
\text { acid transferase, gamm } \\
\text { cium were adjusted. }\end{array}$ & $\begin{array}{l}\text { orous recreational } \\
\text { hoglobin, alkaline } \\
\text { lutamyl transpeptidase, }\end{array}$ \\
\hline
\end{tabular}

The weighted generalised additive models and smooth curve fittings were conducted to characterize the non-linear relationship, and the results further confirmed this positive association (Figs. 2-4).

\section{Discussion}

In this nationally representative sample study, we found that SCre level within the normal range was independently associated with total BMD among adolescents aged 12-19 years. Additionally, this association remained positive in each subgroup stratified by age, gender, or race, and was more prominent in boys than in girls in adolescents aged 12-15 years. 
Muscle is the largest organ in the body that accounts for $40 \%$ of body mass[13]. Muscles grow larger and stronger from birth and start to lose its mass from 25 years of age. The loss in muscle mass and consequently its strength results in sarcopenia[14]. Several evidences suggest that individuals with sarcopenia had lower BMD and a higher risk of osteoporosis[15-17]. SCre concentration may be affected by changes in muscle mass[18]. However, evidence regarding the correlation between SCre and bone health is limited.

To evaluate the correlation of SCre with BMD among older adult with normal renal function, Huh et al.[9] conducted a cross-sectional study using the Fourth Korea NHANES data. Their findings provided the first clinical evidence that low SCre level was independently associated with low BMD. Moreover, their data showed that the positive association between SCre and BMD was more prominent in men than in women. In our study, SCre levels differed by gender. It might be explained by the fact that boys have higher muscle mass than girls. However, after controlling for potential confounding factors, SCre level within the normal range was positively associated with total BMD in adolescents in each subgroup stratified by age, gender, or race. Furthermore, this positive association was more prominent in boys than in girls in adolescents aged 12-15 years. These findings were consistent with the previous study focusing on older adults[9].

It is widely accepted that the increase in peak bone mass achieved during adolescence is effective in preventing osteoporosis and osteoporosis-associated fractures[19]. Creatine has been a popular dietary supplement choice of adolescents, and no study has observed any gastro-intestinal discomforts or changes in markers of clinical health and safety following creatine supplement use periods[20]. Thus, adequate creatine supplementation may be a new strategy for adolescents with low SCre.

By our knowledge, this is the first study that evaluated the association between SCre and BMD in adolescents. Additionally, this nationally representative sample makes our conclusions persuasive and highly relevant to the whole population. However, several weaknesses of our study must be acknowledged. First, due to the cross-sectional nature of NHANES data, it is incapable of indicating the causal association between SCre and BMD. Therefore, a longitudinal follow-up study is required to clarificate the role of creatinine metabolism on bone health. Second, we excluded subjects with abnormal SCre levels, because renal dysfunction may influence BMD [21, 22]. Therefore, our conclusions cannot be used for this special population. Third, other potential confounding factors not included in this study may cause some bias. For example, differences in gender and developmental age during pubertal development is a potential confounding factor in the present study. Hence, we conducted subgroup analyses stratified by age and gender to confirm the results.

In conclusion, our findings indicate that higher SCre levels within the normal range in adolescents aged 12-19 years were associated with higher total BMDs, suggesting that SCre may be a candidate biomarker for bone health in adolescents. It is our hope that this study will stimulate future multidisciplinary research on the effect of creatine and creatinine metabolism on bone health in adolescents. 


\section{Declarations}

\section{Author contributions}

$\mathrm{KYP}$, and HYY contributed to data collection, analysis and writing of the manuscript. ZXZ contributed to study design and editing of the manuscript.

\section{Funding}

This study received no funding.

\section{Consent for publication}

Not applicable.

\section{Competing interests}

The authors declare that they have no competing interests.

\section{Ethical Statement}

The ethics review board of the National Center for Health Statistics approved all NHANES protocols. For participants aged $<18$ years, their parents/guardians provided informed consent, and participants aged $\geq 18$ years provided informed consent on their own.

\section{Acknowledgements}

The authors thank the staff and the participants of the NHANES study for their valuable contributions.

\section{References}

1. Kim A, Baek S, Park S, Shin J. Bone Mineral Density of Femur and Lumbar and the Relation between Fat Mass and Lean Mass of Adolescents: Based on Korea National Health and Nutrition Examination Survey (KNHNES) from 2008 to 2011. Int J Environ Res Public Health 2020;17.

2. Rizzoli R, Bonjour JP. Determinants of peak bone mass and mechanisms of bone loss. Osteoporos Int. 1999;9(Suppl 2):17-23.

3. Golden NH, Abrams SA. Optimizing bone health in children and adolescents. Pediatrics. 2014;134:e1229-43.

4. Pan K, Yao X, Liu M, Zhu Z. Association of Serum Uric Acid Status With Bone Mineral Density in Adolescents Aged 12-19 Years. Front Med (Lausanne). 2020;7:255.

5. Willey JZ, Moon YP, Husain SA, Elkind MSV, Sacco RL, Wolf M, Cheung K, Wright CB, Mohan S. Creatinine versus cystatin $C$ for renal function-based mortality prediction in an elderly cohort: The Northern Manhattan Study. PLoS One. 2020;15:e0226509. 
6. Kreider RB, Kalman DS, Antonio J, Ziegenfuss TN, Wildman R, Collins R, Candow DG, Kleiner SM, Almada AL, Lopez HL. International Society of Sports Nutrition position stand: safety and efficacy of creatine supplementation in exercise, sport, and medicine. J Int Soc Sports Nutr. 2017;14:18.

7. Wyss M, Kaddurah-Daouk R. Creatine and creatinine metabolism. Physiol Rev 2000;80: 1107 - 213.

8. Tabara $Y$, Kohara $K$, Okada $Y$, Ohyagi $Y$, Igase M. Creatinine-to-cystatin $C$ ratio as a marker of skeletal muscle mass in older adults: J-SHIPP study. Clin Nutr. 2020;39:1857-62.

9. Huh JH, Choi SI, Lim JS, Chung CH, Shin JY, Lee MY. Lower Serum Creatinine Is Associated with Low Bone Mineral Density in Subjects without Overt Nephropathy. PLoS One. 2015;10:e0133062.

10. Manual LP, Creatinine NHANES. 2015-2016 https://wwwn.cdc.gov/nchs/data/nhanes/20152016/labmethods/BIOPRO_IMMET_CREATININE_DXC800.pdf.

11. Centers for Disease Control and Prevention. 2018 Centers for Disease Control and Prevention. National Health and Nutrition Examination Survey. https://www.cdc.gov/nchs/nhanes/index.htm (2018), Accessed 17th Oct 2018.

12. von Elm E, Altman DG, Egger M, Pocock SJ, Gotzsche PC, Vandenbroucke JP. The Strengthening the Reporting of Observational Studies in Epidemiology (STROBE) statement: guidelines for reporting observational studies. Lancet. 2007;370:1453-7.

13. Lexell J, Taylor CC, Sjöström M. What is the cause of the ageing atrophy? Total number, size and proportion of different fiber types studied in whole vastus lateralis muscle from 15- to 83-year-old men. J Neurol Sci. 1988;84:275-94.

14. Lo JH, U KP, Yiu T, Ong MT, Lee WY. Sarcopenia: Current treatments and new regenerative therapeutic approaches. J Orthop Translat. 2020;23:38-52.

15. Nielsen BR, Andersen HE, Haddock B, Hovind P, Schwarz P, Suetta C. Prevalence of muscle dysfunction concomitant with osteoporosis in a home-dwelling Danish population aged 65-93 years - The Copenhagen Sarcopenia Study. Exp Gerontol. 2020;138:110974.

16. Kirk B, Phu S, Brennan-OIsen SL, Bani Hassan E, Duque G. Associations between osteoporosis, the severity of sarcopenia and fragility fractures in community-dwelling older adults. Eur Geriatr Med. 2020;11:443-50.

17. Greco EA, Pietschmann P, Migliaccio S. Osteoporosis and Sarcopenia Increase Frailty Syndrome in the Elderly. Front Endocrinol (Lausanne). 2019;10:255.

18. Carnevale V, Tinti MG, Scillitani A, Nieddu L. Estimated Glomerular Filtration Rate and Muscle Mass: Their Relationship in Older Inpatients. J Am Med Dir Assoc. 2019;20:1469-71.

19. Min SK, Oh T, Kim SH, Cho J, Chung HY, Park DH, Kim CS. Position Statement: Exercise Guidelines to Increase Peak Bone Mass in Adolescents. J Bone Metab. 2019;26:225-39.

20. Jagim AR, Stecker RA, Harty PS, Erickson JL, Kerksick CM. Safety of Creatine Supplementation in Active Adolescents and Youth: A Brief Review. Front Nutr. 2018;5:115.

21. Chen H, Lips P, Vervloet MG, van Schoor NM, de Jongh RT. Association of renal function with bone mineral density and fracture risk in the Longitudinal Aging Study Amsterdam. Osteoporos Int. 
2018;29:2129-38.

22. Han W, Bai XJ, Han LL, Akhtari S, Sun XF, Chen XM. Association between the age-related decline in renal function and lumbar spine bone mineral density in healthy Chinese postmenopausal women. Menopause. 2018;25:538-45.

\section{Figures}

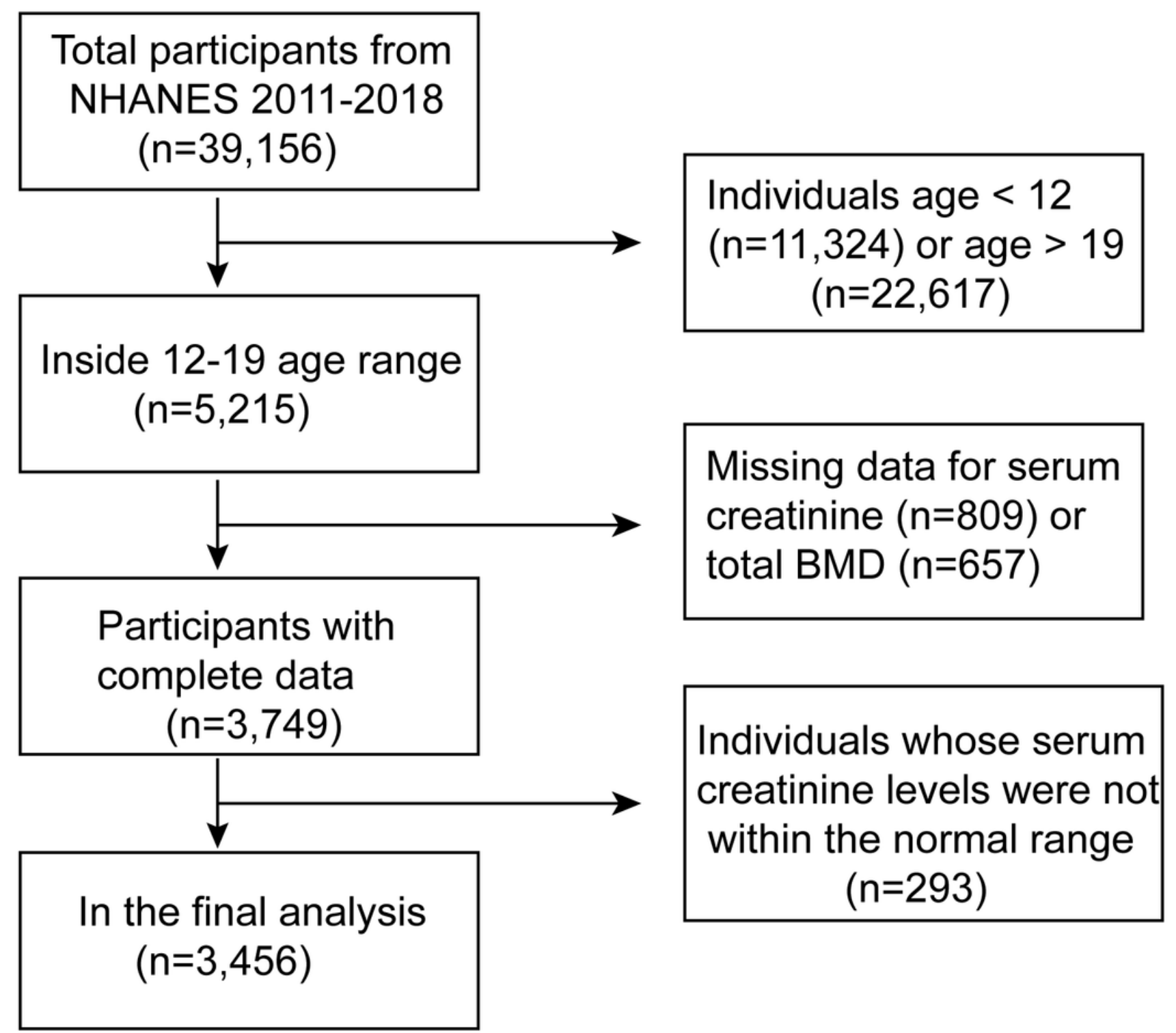

Figure 1

The flow chart of the participants. 
(a)

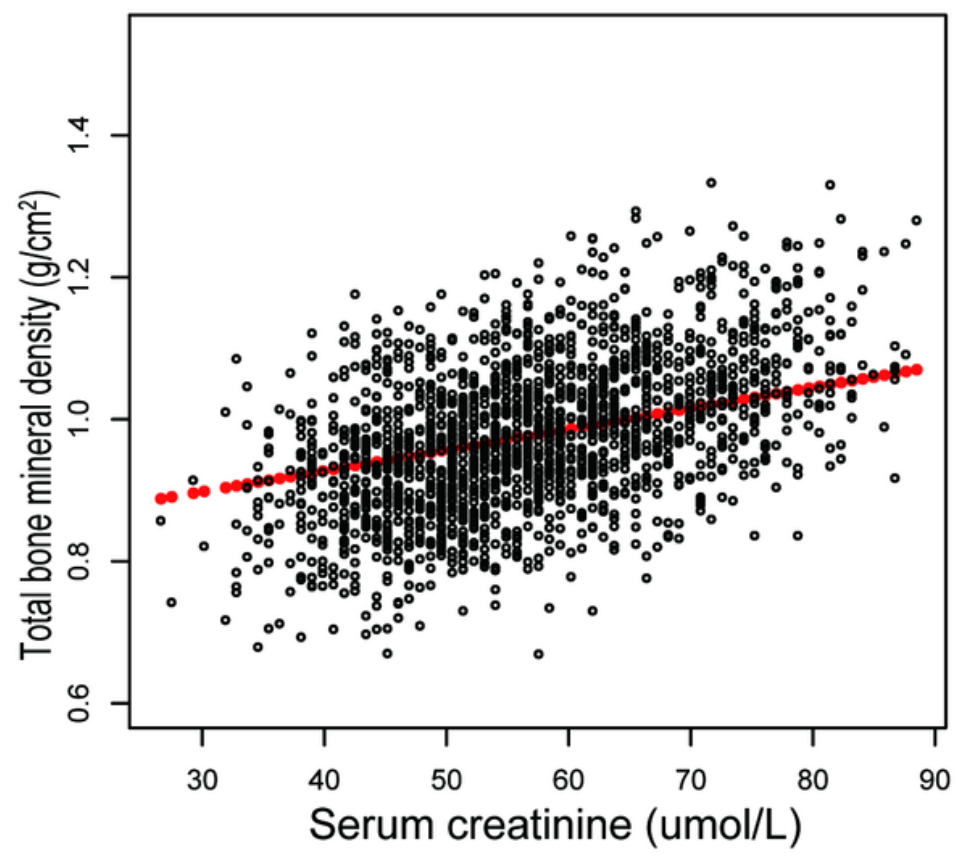

(b)

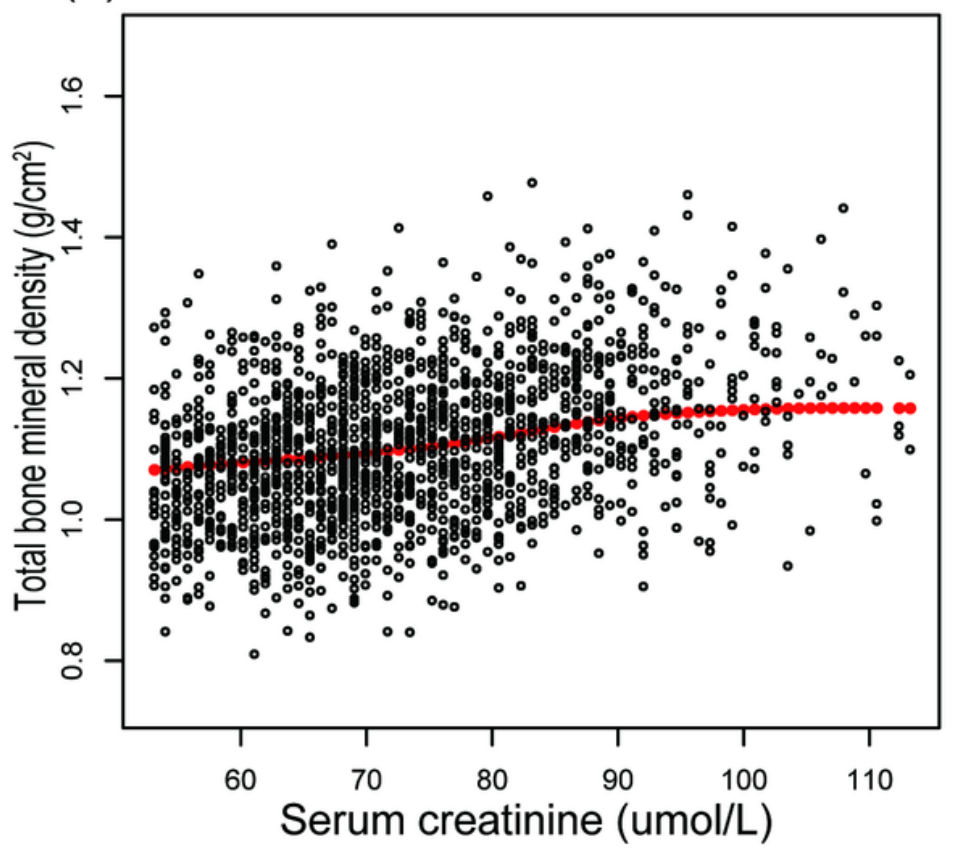

Figure 2

Association between serum creatinine and total bone mineral density. (a) 12-15 years (b)16-19 years Circles represent individual data points. Sample weighted regressions were adjusted for age, gender, race, body mass index, ratio of family income to poverty, vigorous recreational activities, blood urea nitrogen, total protein, total cholesterol, serum glycohemoglobin, alkaline phosphatase, alanine amino transferase, aspartic acid transferase, gamma-glutamyl transpeptidase, serum uric acid, serum phosphorus, and serum calcium.

(a)

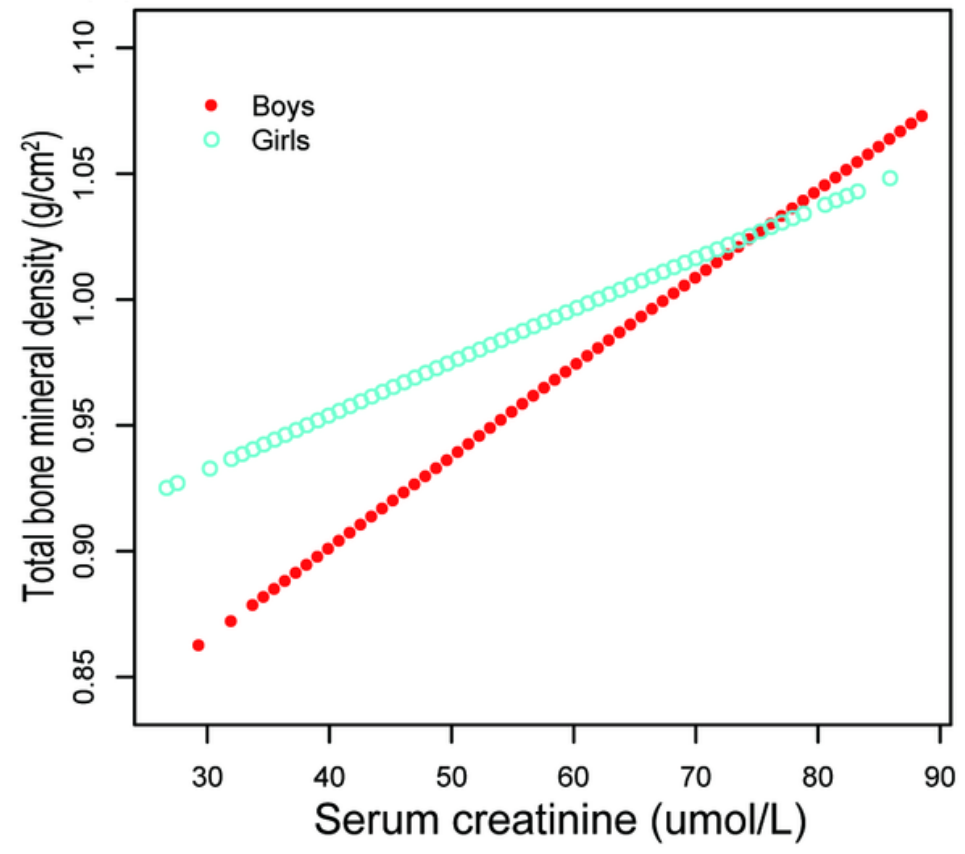

(b)

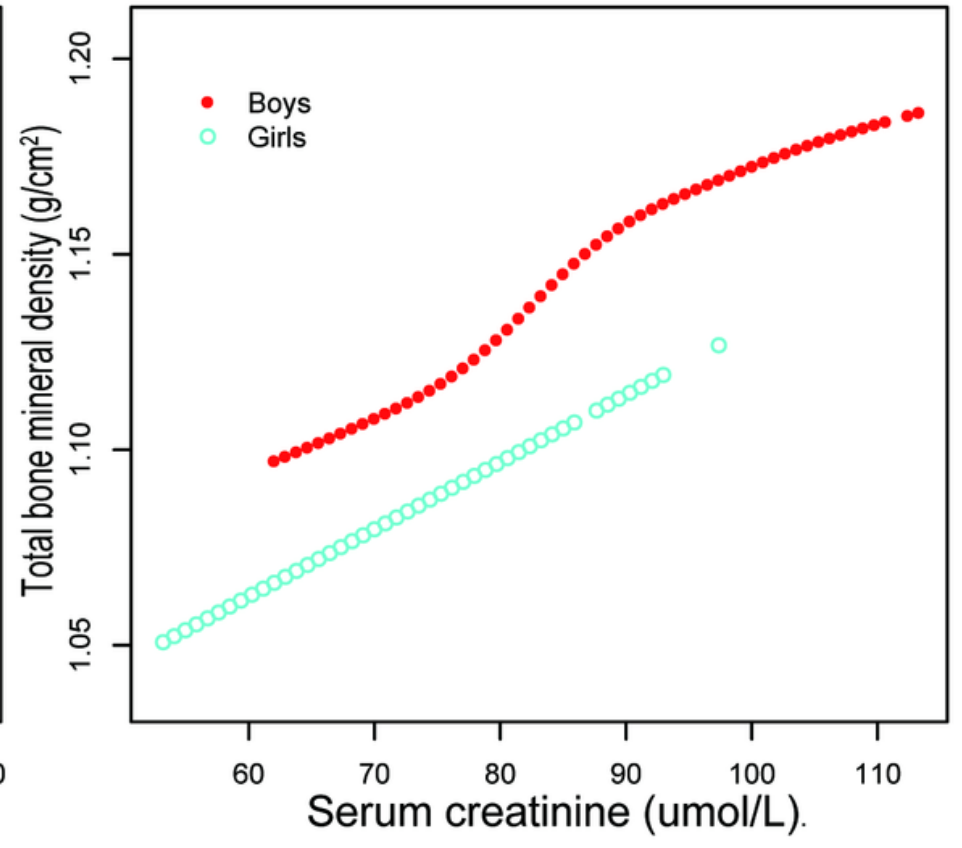




\section{Figure 3}

Association between serum creatinine and total bone mineral density, stratified by gender. (a) 12-15 years (b) 16-19 years Sample weighted regressions were adjusted for age, race, body mass index, ratio of family income to poverty, vigorous recreational activities, blood urea nitrogen, total protein, total cholesterol, serum glycohemoglobin, alkaline phosphatase, alanine amino transferase, aspartic acid transferase, gamma-glutamyl transpeptidase, serum uric acid, serum phosphorus, and serum calcium.

(a)

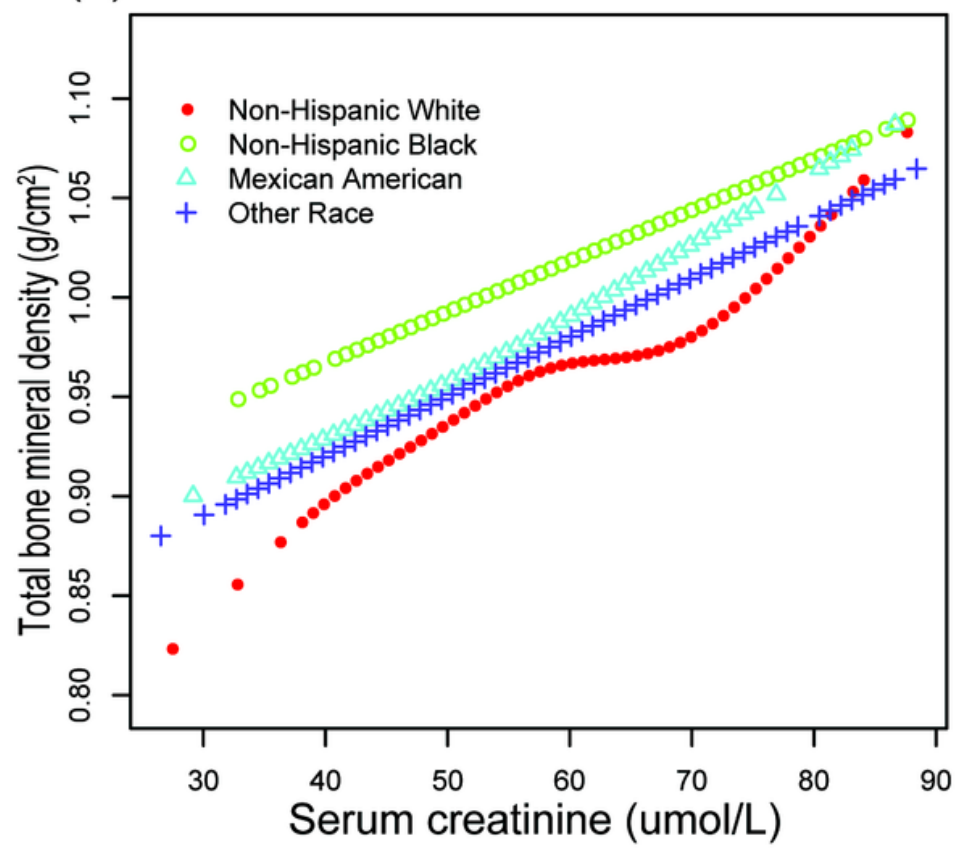

(b)

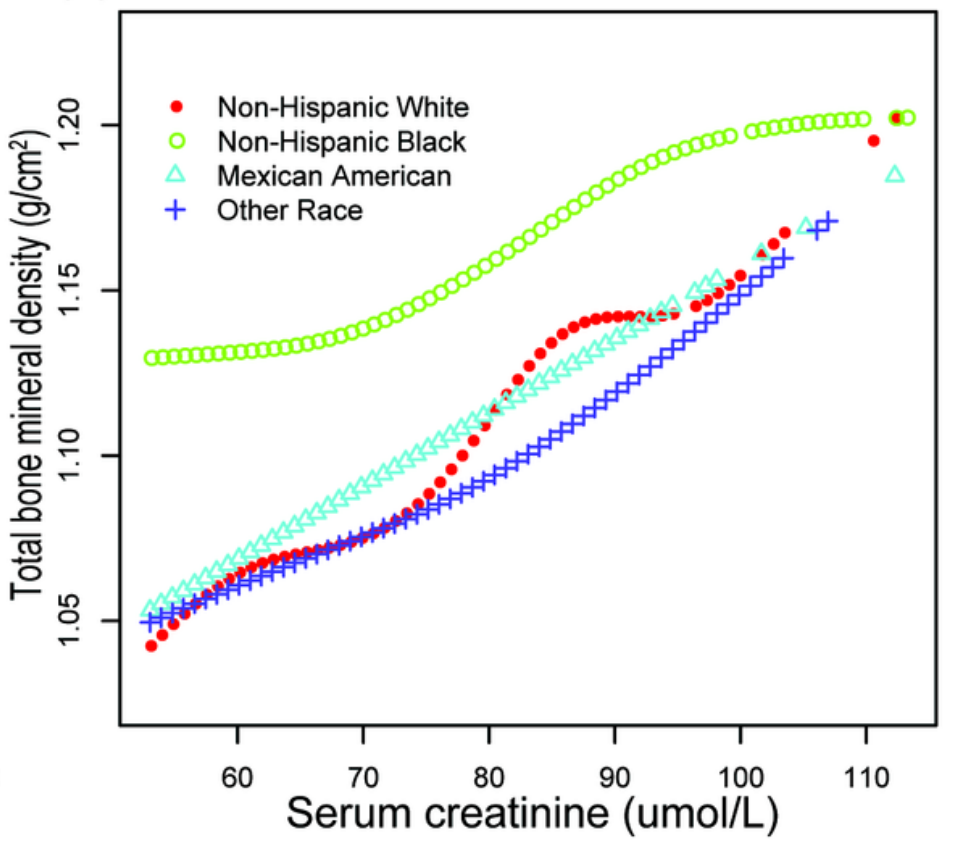

\section{Figure 4}

Association between serum creatinine and total bone mineral density, stratified by race. (a) 12-15 years (b) 16-19 years Sample weighted regressions were adjusted for age, gender, body mass index, ratio of family income to poverty, vigorous recreational activities, blood urea nitrogen, total protein, total cholesterol, serum glycohemoglobin, alkaline phosphatase, alanine amino transferase, aspartic acid transferase, gamma-glutamyl transpeptidase, serum uric acid, serum phosphorus, and serum calcium. 